\title{
Herald bleeding from a ruptured primary hepatic angiosarcoma: A case report
}

\author{
SHAMIR O. CAWICH and CHUNILAL RAMJIT \\ Department of Clinical Surgical Sciences, University of the West Indies, St. Augustine Campus, Trinidad and Tobago
}

Received January 21, 2015; Accepted February 20, 2015

DOI: $10.3892 / \mathrm{mco} .2015 .575$

\begin{abstract}
Primary hepatic angiosarcomas (PHAs) are rare tumours with an estimated annual incidence of 0.05 per million in the Caribbean, which is similar to that reported in Western countries. Although a number of cases are detected post-mortem, the most common clinical presentation is with tumour rupture and herald bleeding. This is the case report of a 60-year old female patient who presented with vague upper abdominal pain, found via imaging examinations to be due to a ruptured hepatic tumour in segment III of the liver. The tumour was removed via laparoscopic left lateral sectionectomy, with clear resection margins. The histopathological and immunohistochemical examination established the diagnosis of PHA. Therefore, an index of suspicion should be maintained in selected patients and, when detected ante-mortem, PHAs should be treated aggressively with complete surgical resection to achieve microscopically clear margins, as the response of these tumours to other forms of adjuvant therapy may be unpredictable.
\end{abstract}

\section{Introduction}

Primary hepatic angiosarcoma (PHA) is an aggressive malignant neoplasm arising from the endothelium of blood vessels within the liver (1). PHA is rarely encountered in Western countries (2-4), where it reportedly accounts for $0.6 \%$ (1) to $2 \%$ (4) of all primary liver tumours.

Due to its aggressive growth patterns and indistinct clinical characteristics, the diagnosis of PHA is often made post-mortem $(1,4)$. Approximately 1 in 5 patients are diagnosed in vivo when they present with capsular rupture and intraperitoneal bleeding $(2,4,5)$; these tumours, however, tend to be locally advanced when diagnosed ante-mortem (4).

Correspondence to: Dr Shamir O. Cawich, Department of Clinical Surgical Sciences, University of the West Indies, St. Augustine Campus, Trinidad and Tobago

E-mail: socawich@hotmail.com

Key words: angiosarcoma, hepatectomy, liver, rupture, hemoperitoneum
This is the case report of a 60 -year old female patient who presented with a ruptured hepatic tumour histopathologically diagnosed as PHA and a review of the clinicopathological characteristics of PHAs.

\section{Case report}

A 60-year old woman with no known chronic medical conditions experienced vague upper abdominal pain for 6 weeks prior to presentation. The patient reported no weight loss, anorexia, gastrointestinal symptoms or history of trauma. The physical examination revealed no abnormalities.

The blood tests revealed a haemoglobin count of 10,000/dl. The electrolyte levels and the renal and liver function tests were normal. An endoscopic evaluation excluded the presence of gastrointestinal lesions. However, the abdominal ultrasound revealed the presence of free intraperitoneal fluid and a tumour in segment III of the liver. Contrast-enhanced computed tomography scans of the chest, abdomen and pelvis confirmed the presence of perihepatic fluid and the presence of a heterogenous 4-cm tumour in segment III of the liver. The remaining segments of the liver were normal, with no evidence of metastatic disease.

The patient was put under general anaesthesia and underwent laparoscopic liver resection. There was no evidence of metastatic disease in the peritoneal cavity and the tumour was readily identified by its bosselated surface (Fig. 1). Laparoscopic ultrasonography was used to achieve clear resection margins (Fig. 2). A laparoscopic left lateral sectionectomy was completed uneventfully.

The pathological examination confirmed the presence of a tumour sized $3 \times 4 \times 2.5 \mathrm{~cm}$, with a focal point at which Glisson's capsule was breached (Fig. 3). The resection margins were clear of tumour for $>2 \mathrm{~cm}$. Histologically, the tumour was composed of sheets of poorly differentiated neoplastic cells (Fig. 4). The malignant cells were spindle-shaped and possessed hyperchromatic nuclei, indistinct cell borders and scant cytoplasm (Fig. 5). Significant abnormal mitotic activity was observed and bizarre multinucleated giant cells were present, particularly at the periphery of the lesion (Fig. 6). The tumour characteristically grew along the hepatic sinusoids and around residual hyperplastic hepatocytes, which were otherwise normal. There were scattered islands of haematopoietic cells and large areas of thrombosis and infarction, with accompanying tumour necrosis. The histological appearance 


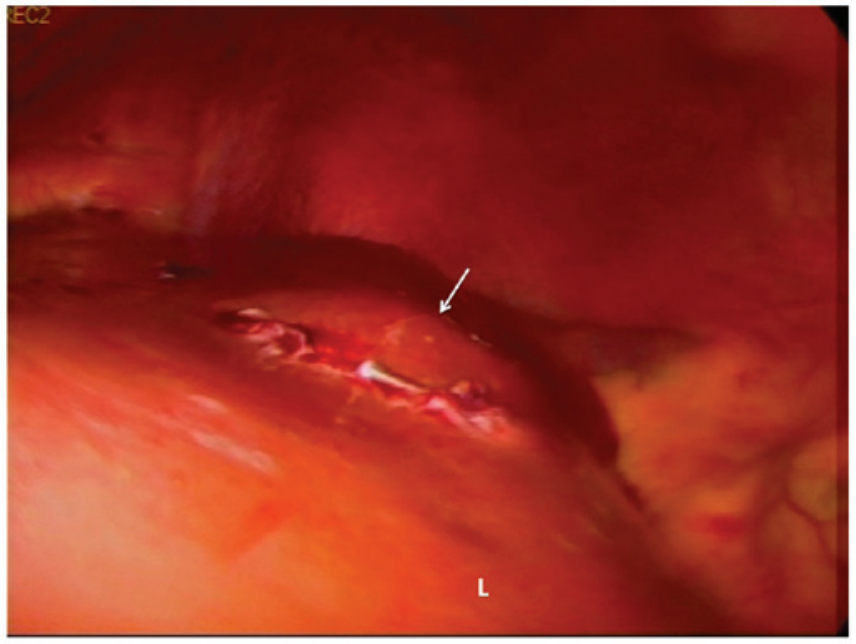

Figure 1. Laparoscopic view of the left liver. The elevated bosselated surface of the tumour (arrow) is easily be distinguished from the normal liver surface (L) covered by the Glisson's capsule.

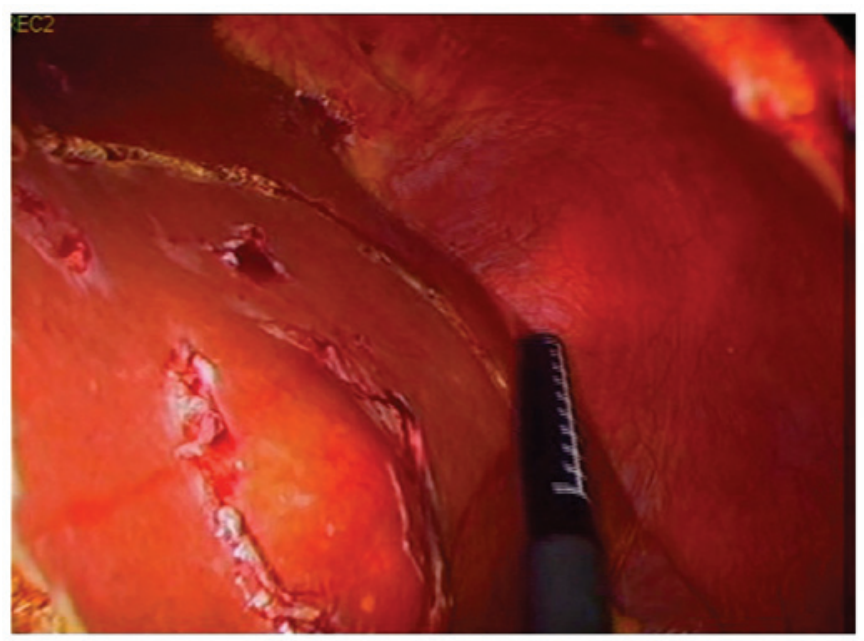

Figure 2. Laparoscopic view of the left liver. A laparoscopic ultrasound probe is used to delineate the tumour in order to achieve clear resection margins.

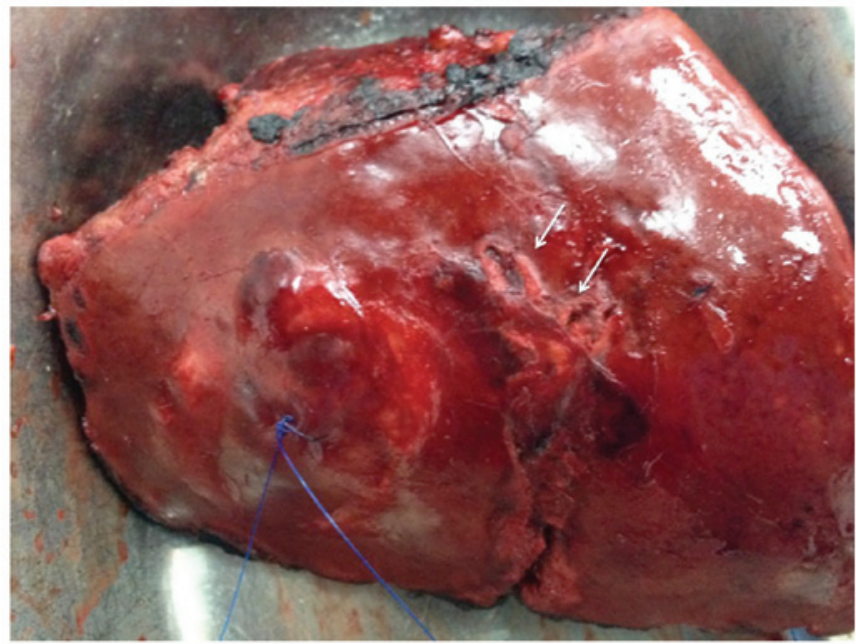

Figure 3. Excised hepatic segment III demonstrating the bosselated surface of the tumour (blue suture) and the area of capsular breach (arrows), which was responsible for the herald bleed.

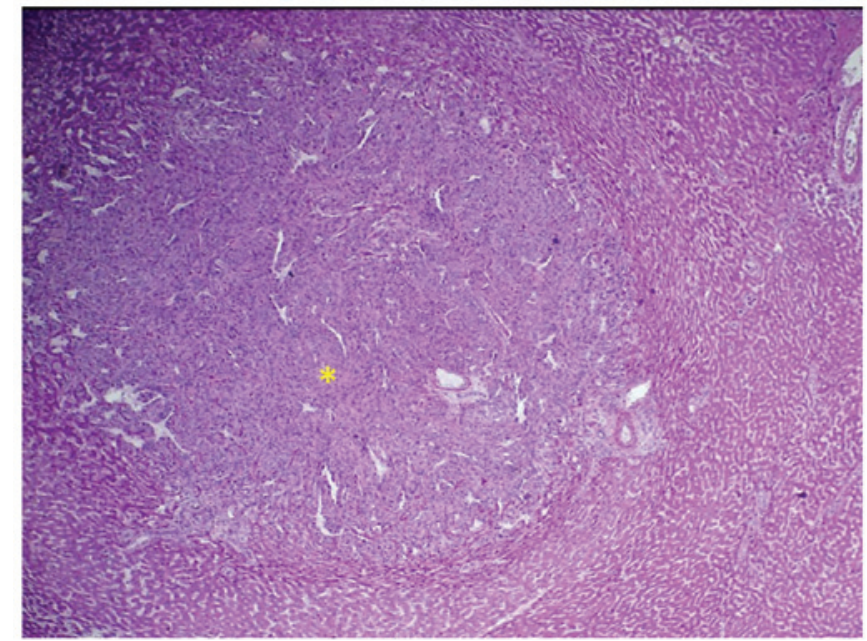

Figure 4. Low-power (x10) view of a tumour nodule (yellow asterisk placed in the centre) composed of sheets of neoplastic cells surrounded by normal liver.

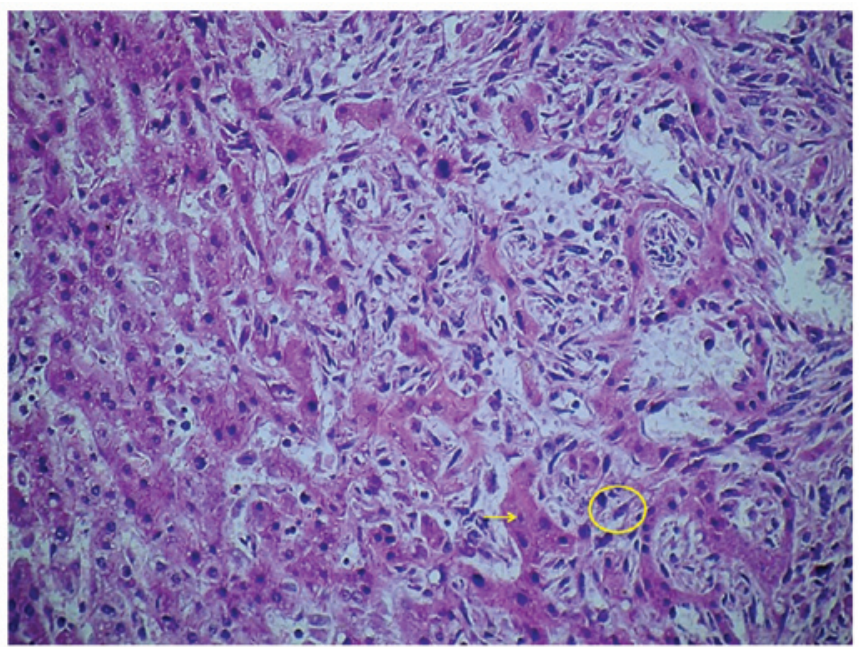

Figure 5. High-power (x20) view of spinde-shaped malignant cells with hyperchromatic nuclei and scant cytoplasm (a single neoplastic cell is encircled in the figure). The malignant cells may be seen dissecting between the normal hepatic plates (yellow arrow).

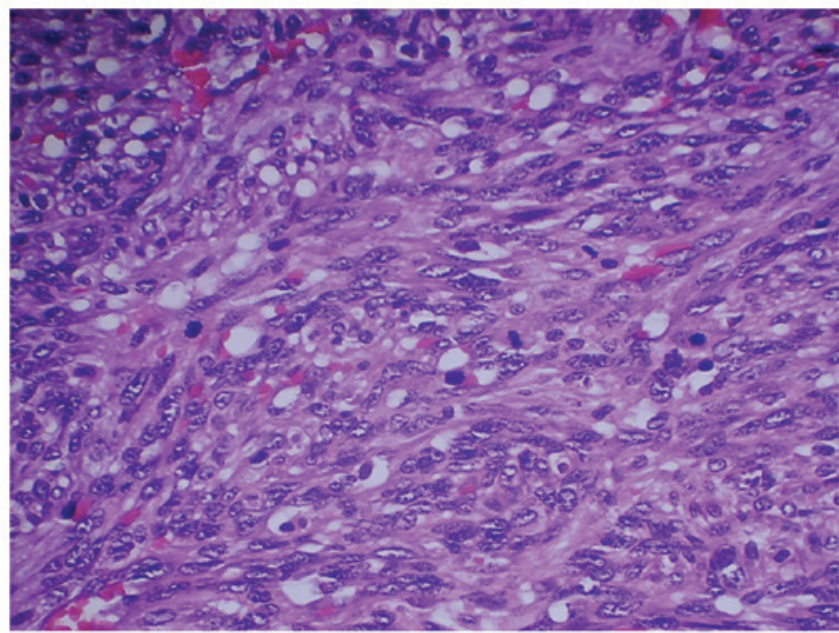

Figure 6. A slide of the tumour (magnification, x100), showing bizzare disordered malignant cells with undifferentiated cytomorphology. The cells exhibit hyperchromatic nuclei, scant cytoplasm and indistinct borders. 
indicated a high-grade pleomorphic sarcoma consistent with PHA. The diagnosis was verified by immunohistochemistry, which was positive for CD31 and factor VIII (FVIII)-R antigen, but negative for desmin, keratin, S-100 protein and discovered on GIST-1.

\section{Discussion}

Angiosarcomas are aggressive malignant neoplasms originating from the endothelium of blood vessels (2). The liver is the fifth most common site of origin for angiosarcomas (2-3). Although it accounts for $0.6 \%$ (1) to $2 \%$ (4) of primary liver tumours in Western countries, PHA is a rare occurrence (1-4). The three largest published series are audits from national sarcoma registries, with a limited number of cases, namely 6 cases over 15 years in China, 26 cases over 19 years in Taiwan (6) and 32 cases over 16 years in Britain (7).

In the largest reported series of 32 cases from the British Hepatic Angiosarcoma Register, Baxter (7) reported the annual incidence of PHA to be 0.16 cases per million persons. An annual incidence as high as 0.26 per million was reported in an audit from the United States by Vianna et al (8). There has been no prior report on PHA from the Anglophone Caribbean, a region consisting of 17 English-speaking Caribbean countries with a cumulative population of 6.5 million (9). We commenced a hepatobiliary registry to record all primary and metastatic liver tumours encountered in the three main referral centres for the Anglophone Caribbean in January, 2011 (9). This was the first case encountered in the registry, allowing us to estimate the annual incidence as 0.05 per million population in the Anglophone Caribbean, which is comparable to the incidence in Western countries $(7,8)$.

Our patient was a 60-year old woman; this is the usual age at which a PHA is diagnosed (4), although they are more common in men $(4,5,7)$. The reported male:female ratio ranges from 1.9:1 in Taiwan (7) to as high as 4:1 in Britain (8).

We were unable to identify an aetiological factor in our patient. Early studies suggested that environmental exposure to Thorotrast $(4,7)$, vinyl (7), polyvinyl chloride-manufacturing materials (7), arsenic, pesticides and radium (4) were risk factors for PHA; however, exposure to these agents has become uncommon in modern practice. It was not surprising, therefore, that neither of these environmental risk factors were present in our case.

PHAs are often recognized only at autopsy $(4,10)$, as the diagnosis is often missed in vivo when patients report vague, non-specific symptoms and the tumour exhibits aggressive growth patterns $(2,4,10,11)$. When the diagnosis is made ante-mortem, PHAs are usually identified at an advanced stage, when therapeutic options are limited $(4,12,13)$. In our case, the diagnosis was made relatively early in the absence of metastatic or locally advanced disease.

The patient presented after the tumour had ruptured. Intraperitoneal haemorrhage following tumour rupture appears to be a relatively common method of acute presentation $(2,4,5,14,15)$, usually accompanied by a high mortality risk due to exsanguination $(2,4,15)$. Survivors usually present with a herald bleed, as in our case. This presentation accounts for $22 \%$ (4) to $27 \%$ (10) of the cases diagnosed ante-mortem.
The histological characteristics observed in this case were typical of PHA. Normal hepatocytes were observed, with infiltrating islands of neoplastic endothelial cells extending along sinusoidal vascular channels $(2,4)$. The individual neoplastic cells are often anaplastic or spindle-shaped, with pale cytoplasm, pleomorphic nuclei and indistinct borders (4). The presence of bizzare giant cells and epithelioid cell patterns, as seen in this case, are also characteristic (2). The cells stained positive for CD31 and FVIII, which are characteristic of PHA $(2,4)$. Although not present in this case, PHA cells may also stain positive for CD34 $(2,4)$ and vimentin $(2)$.

PHAs are aggressive tumours. Without treatment, the median survival is reported to be only 6 months from the time of diagnosis $(2,4,10,16)$. As the response to systemic chemotherapy and radiotherapy is unpredictable $(2,17)$, it is crucial for surgeons to achieve complete resection with clear margins. However, even following complete (R0) resection, long-term survival is poor, with existing 5-year survival rate estimates of $3 \%(2,4,10,16)$.

In conclusion, PHAs are rare tumours, with an estimated annual incidence of 0.05 per million in the Caribbean. Intraperitoneal haemorrhage from tumour rupture appears to be a common method of presentation. Patients with herald bleeds should be urgently treated, prior to the development of exsanguinating haemorrhage, by hepatobiliary surgeons performing aggressive resection to ensure clear margins.

\section{References}

1. Molina E and Hernandez A: Clinical manifestations of primary hepatic angiosarcoma. Dig Dis Sci 48: 677-682, 2003.

2. Chien CY, Hwang CC, Yeh CN, Chen HY, Wu JT, Cheung CS Lin CL, Yen CL, Wang WY and Chiang KC: Liver angiosarcoma, a rare liver malignancy, presented with intraabdominal bleeding due to rupture - a case report. World J Surg Oncol 10: 23, 2012.

3. Young RJ, Brown NJ, Reed MW, Hughes D and Woll PJ: Angiosarcoma. Lancet Oncol 11: 983-991, 2010.

4. Huang NC, Wann SR, Chang HT, Lin SL, Wang JS and Guo HR: Arsenic, vinyl chloride, viral hepatitis, and hepatic angiosarcoma: A hospital-based study and review of literature in Taiwan. BMC Gastroenterol 11: 142, 2011.

5. Ho S-Y, Tsai C-C, Tsai Y-C and Guo H-R: Hepatic angiosarcoma presenting as hepatic rupture in a patient with long-term ingestion of arsenic. J Formos Med Assoc 103: 374-379, 2004

6. Wang TH, Pan ZG and Ren ZG: Rare primary liver malignant tumor. Fudan Univ J Med Sci 36: 221-224, 2009.

7. Baxter PJ: The British hepatic angiosarcoma register. Environ Health Perspect 41: 115-116, 1981.

8. Vianna NJ, Brady JA and Cardamone AT: Epidemiology of angiosarcoma of liver in New York State. N Y State J Med 81: 895-899, 1981.

9. Cawich SO, Johnson PB, Shah S, et el: Overcoming obstacles to establish a multidisciplinary team approach to hepatobiliary diseases: a working model in a Caribbean setting. J Multidiscip Healthc 7: 227-230, 2014.

10. Locker GY, Doroshow JH, Zwelling LA and Chabner BA: The clinical features of hepatic angiosarcoma: A report of four cases and a review of the English literature. Medicine (Baltimore) 58: 48-64, 1979.

11. Timaran $\mathrm{CH}$, Grandas $\mathrm{OH}$ and Bell JL: Hepatic angiosarcoma: Long-term survival after complete surgical removal. Am Surg 66: 1153-1157, 2000.

12. Poggio JL, Nagorney DM, Nascimento AG, Rowland C, Kay P, Young RM and Donohue JH: Surgical treatment of adult primary hepatic sarcoma. Br J Surg 87: 1500-1505, 2000.

13. Jaffe BM, Donegan WL, Watson F and Spratt JS Jr: Factors influencing survival in patients with untreated hepatic metastases. Surg Gynecol Obstet 127: 1-11, 1968.

14. Tsai CC, Hsieh JF, Han SJ and Mo LR: Hemoperitoneum secondary to biopsy of the hepatic angiosarcoma. Chin J Radiol 24: 37-40, 1999. 
15. Lee SW, Song CY, Gi YH, Kang SB, Kim YS, Nam SW, Lee DS and Kim JO: Hepatic angiosarcoma manifested as recurrent hemoperitoneum. World J Gastroenterol 14: 2935-2938, 2008.

16. Falk H, Herbert JT, Edmonds L, Heath CW Jr, Thomas LB and Popper H: Review of four cases of childhood hepatic angiosarcoma - elevated environmental arsenic exposure in one case. Cancer 47: 382-391, 1981.
17. Almogy G, Lieberman S, Gips M, Pappo O, Edden Y, Jurim O, Simon Slasky B, Uzieli B and Eid A: Clinical outcomes of surgical resections for primary liver sarcoma in adults: Results from a single centre. Eur J Surg Oncol 30: 421-427, 2004. 